\title{
Stereoscopic watermarking by horizontal noise mean shifting
}

\author{
Ji-Won Lee, Hee-Dong Kim, Hak-Yeol Choi, Sung-Hee Choi, and Heung-Kyu Lee \\ Department of Computer Science, Korea Advanced Institute of Science and Technology, \\ 291 Daehak-ro, Yuseong-gu, Daejeon, Republic of Korea
}

\begin{abstract}
Depth-image-based rendering (DIBR) is a method to represent a stereoscopic content. The DIBR consists of a monoscopic center view and an associated per-pixel depth map. Using these two components and given depth condition from a user, the DIBR renders left and right views. The advantages of DIBR are numerous. The user can choose not only the monoscopic or stereoscopic view selectively, but also the depth condition what he prefers when he watches a stereoscopic content. However, in the view of copyright protection, since not only the center view but also each left or right view can be used as a monoscopic content when they are illegally distributed, the watermark signal which is embedded in the center view must have an ability to protect the respective three views. In this study, we solve this problem by exploiting the horizontal noise mean shifting (HNMS) technique. We exploit the fact that the objects in the view are shifted only to horizontal way when the center view renders to the left and right views. Using this fact, the proposed stereoscopic watermarking scheme moves the mean of horizontal noise histogram which is invariant to horizontal shifting, and we achieve good performance as shown in the experimental results.
\end{abstract}

Keywords: Stereoscopic watermarking, Depth-Image-Based Rendering (DIBR), 2D-3D conversion, Horizontal noise mean shifting

\section{INTRODUCTION}

As the demand of stereoscopic contents increases rapidly, many stereoscopic contents are generated by content providers. These stereoscopic contents can be represented in various formats. Among them, two representation formats for three dimensional (3D) contents are used widely: the stereo image recording (SIR) and the DIBR. ${ }^{1}$ The SIR stores both left and right views directly. Thus, a 3D content that is represented in the SIR is easy to store and play. However, if the user wants to watch monoscopic contents, the SIR has to use the left or right view, not the center view. Further, since the SIR doesn't store the depth information, the stereoscopic content formatted in the SIR could not change the depth environment. Another drawback of the SIR is the coding inefficiency. The transmission bandwidth of SIR formatted stereoscopic contents is needed as twice as larger than that of monoscopic contents. The DIBR consists of a monoscopic center view and an associated per-pixel depth map. Using these two components and given depth information from the user, the DIBR renders to left and right views. Since human eyes are insensitive to small changes of depth level, a depth map can be compressed more strongly than a monoscopic view. Thus, the coding efficiency is better than that of SIR. Also, since stereoscopic view of DIBR formatted contents is generated by rendering to the left and right views in the device using the center view and depth map, users can control the depth condition of the stereoscopic view that they prefer. Further, users can watch $2 \mathrm{D}$ vision to $3 \mathrm{D}$ vision selectively because the DIBR has a center view. Because of these numerous advantages, the DIBR is in the limelight nowadays.

However, copyright protection of DIBR formatted contents is difficult. Since not only the center view but also warped left or right view can be used as monoscopic contents when one of these views is illegally distributed, we have to protect all three views. The important thing is that two different left, right view sets rendered from one DIBR formatted content could be different if the rendering depth conditions are different. In other words, the stereoscopic watermarking technique which embeds the watermark signal only into the center view only and can detect the embedded copyright information from not only the center view but also left and right views warped from any depth condition without helping the depth map is needed.

There are few watermarking techniques for DIBR formatted contents. ${ }^{2,3}$ In case of Lee's study, ${ }^{2}$ they embedded a watermark signal into the center view by exploiting spatial and temporal factors for imperceptibility.

Media Watermarking, Security, and Forensics 2012, edited by Nasir D. Memon, Adnan M. Alattar, Edward J. Delp III, Proc. of SPIE Vol. 8303, 830307 · @ 2012 SPIE · CCC code: 0277-786X/12/\$18 · doi: 10.1117/12.912075 


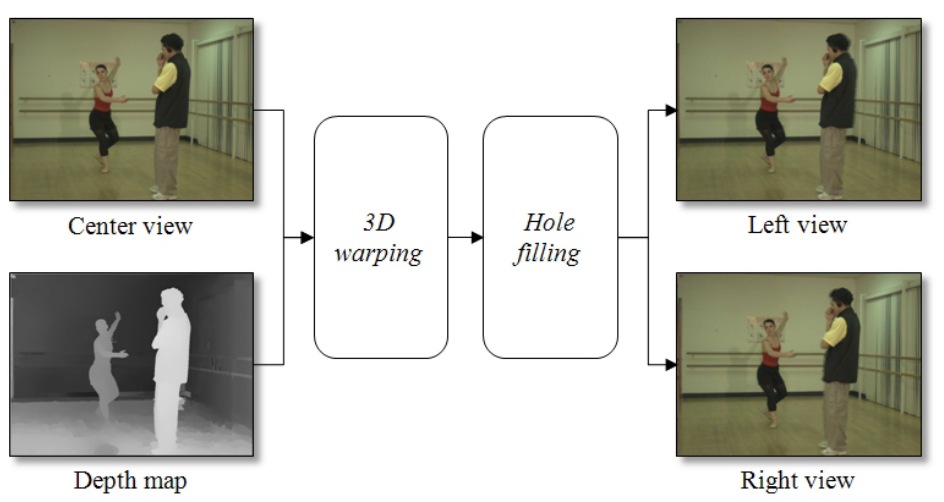

Figure 1. Left and right virtual view generation process

Since the main concern of this scheme is visual quality of a stereoscopic view, it has very good imperceptibility when the watermarked center view is warped to a stereoscopic view. However, they are only interested in the copyright of a center view, so that the embedded information is lost in the warped left and right views. Lin el al. ${ }^{3}$ considered 2D-3D conversion. Since this method embedded three watermarks for center, left, and right views into the center view by pre-calculating the shifting distance after warping using the preset depth condition, they acquired the robustness to 2D-3D conversion. However, the visual quality of this method is not relatively good because they embedded three watermarks into the center view simultaneously. Further, since they fixed the depth condition, this method works only already set shifting distance of left and right views. Nevertheless, Lin's study is meaningful because they first considered the stereoscopic watermark technique robust to 2D-3D conversion.

$2 \mathrm{D}$ to $3 \mathrm{D}$ warping process can be viewed as a local translation on $x$-axis. It means the objects are moved only horizontally by $2 \mathrm{D}$ to $3 \mathrm{D}$ warping process. In this study, we propose the stereoscopic watermarking method by shifting the mean of horizontal noise histogram. Since the mean of horizontal noise histogram is invariant to the local horizontal shifting, the proposed scheme is invariant to 2D-3D conversion. Also, since the proposed scheme embeds the watermark signal by exploiting the human visual system, we achieve high visual quality as well.

The remainder of this paper is organized as follow. In Sec. 2, DIBR system which is the target system of the proposed watermarking scheme is introduced. In Sec. 3, we propose embedding and detection process of the proposed scheme. In Sec. 4, we present experimental results to show the performance of the proposed scheme. Finally, Sec. 5 provides concluding remarks.

\section{DIBR SYSTEM}

As shown in fig. 1, the left and right views can be rendered with a color image, which is typically a center view, and a depth map corresponding to the color image. Depth maps, which have gray scale range from 0 to 255 , are normally generated by special purpose cameras or depth estimation techniques from 2D images. Relatively nearer points to viewers have values close to 255, while farther points have darker pixel value around 0 .

Using depth map, the center view is warped into left and right views by formula defined below. ${ }^{3}$

$$
\begin{aligned}
& x_{L}=x+\left(\frac{t_{x}}{2} \times \frac{f}{Z}\right) \\
& x_{R}=x-\left(\frac{t_{x}}{2} \times \frac{f}{Z}\right)
\end{aligned}
$$

Here, $x_{R}$ and $x_{L}$ are the coordinates after 3D warping from the original coordinate $x$ of center view. $f$ is the focal length of camera, $t_{x}$ is the baseline distance. $Z$ is the relative depth calculated by

$$
Z=\frac{d}{255}\left(Z_{\text {near }}-Z_{\text {far }}\right)+Z_{\text {far }}
$$




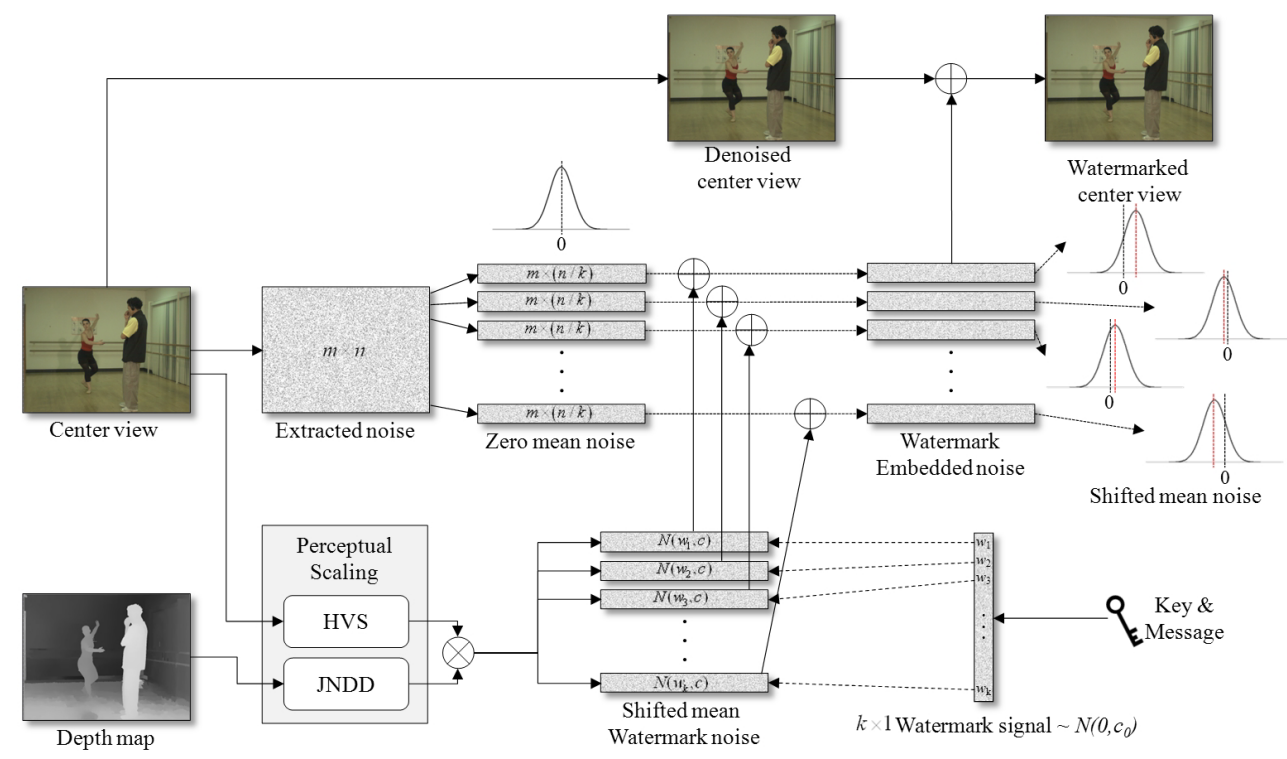

Figure 2. Overview of embedding process

where $d$ is the depth value corresponding to center view pixel. $Z_{\text {near }}$ and $Z_{\text {far }}$ mean the range of the depth value in front and behind of the screen, respectively. The point needed to attention from the 3D warping process is that pixels in a center view are only moved to horizontal way.

After 3D warping process, the left and right views still have a problem of holes since some parts in left and right views were occluded in the center view and there was no information to restore holes correctly from the center view. To solve this problem, many hole filling algorithms are proposed. ${ }^{1,4}$ Basically, holes are recovered by interpolation operations using adjacent pixels.

\section{THE WATERMARKING SCHEME}

Since not only the center view, which is basic component of DIBR, but also the left or right view, which is warped from the DIBR, can be used as $2 \mathrm{D}$ contents, we have to prevent each three views from illegal distribution. To protect these views, we propose a stereoscopic watermarking scheme that is robust to 2D-3D conversion under various depth conditions in DIBR system. We exploit two principles to design this scheme;

- The noise histogram of certain image follows zero mean Laplace distribution.

- When a center view is warped to the left and right views, the objects inside the view are only moved horizontally.

The detail embedding and detection process is described in the next two subsections, and its false positive rate is analyzed in the third subsection.

\subsection{Embedding process}

The proposed scheme embeds a watermark signal into the center view by shifting the mean of noise histogram. Fig. 2 shows the overview of embedding process. To embed the watermark signal, we first extract the noise image from the center view whose size is $m \times n$. Then, we horizontally slice the extracted noise image up the $k$ noise bars whose size are $m \times(n / k)$, where $k$ is the dimension of watermark signal and $k \leq n$. The reference watermark signal $w_{r}$, follows Gaussian distribution with zero mean and constant variance $c_{0}$, is generated using a secret key. Since each noise pixel follows zero mean Laplace distribution, each extracted noise bar follows zero mean Laplace distribution as well. To embed the $i^{t h}$ element of $w_{r}$, denoted as $w_{r}(i)$, into the $i^{t h}$ noise bar, we 
add the shifted mean watermark noise to the $i^{\text {th }}$ extracted noise bar. Here, the mean of shifted mean watermark noise is the same as $i^{t h}$ element of watermark signal. The shifted mean watermark noise is calculated as follow.

$$
S W_{i}(x, y)=\left(N^{(0, c)}(x, y)+w_{r}(i)\right) \cdot \psi(x, y) .
$$

Here, $S W_{i}(x, y)$ is the $i^{t h}$ shifted mean watermark noise and $0<x \leq m, 0<y \leq n / k . N^{(0, c)}(x, y)$ is a random variable that follows Gaussian distribution with zero mean and variance $c$. The perceptual scaling factor $\psi(x, y)$ is calculated by multiplying of

$$
\psi(x, y)=\eta(x, y) \cdot \kappa(x, y) .
$$

Here, $\eta(x, y)$ is a local weighting factor of the pixel from noise visibility function (NVF) ${ }^{5}$ and $\kappa(x, y)$ is a local weighting factor using the just noticeable depth difference (JNDD). NVF utilizes the fact that human eyes are difficult to perceive the noise in the textured regions and edges. The formula for NVF is as follow.

$$
n v f(x, y)=\frac{1}{1+\theta \sigma_{l}^{2}(x, y)}
$$

with

$$
\theta=\frac{D_{n v f}}{\sigma_{l_{\max }}^{2}}
$$

Here, $\sigma_{l}^{2}(x, y)$ is local variance of the image in a window, whose size is $l \times l$, centered on the pixel with coordinates $(x, y), 1 \leq x \leq m, 1 \leq y \leq n$. $\theta$ is a tuning parameter which must be chosen for every particular image. $\sigma_{l_{\max }}^{2}$ is the maximum local variance for a given image. $D_{n v f} \in[50,100]$ is a parameter which is determined experimentally. $n v f(x, y)$ approaches 1 in the flat regions, whereas, $n v f(x, y)$ approaches 0 in the textured regions and edges. The local weighting factor $\eta(x, y)$ is calculated using $n v f(x, y)$ and two scaling factors $S_{0}$ and $S_{1}$.

$$
\eta(x, y)=S_{0}+\left(S_{1}-S_{0}\right) \cdot \operatorname{nvf}(x, y) .
$$

In our embedding scheme, the size $l \times l$ for local variance $\sigma_{l}^{2}$ of NVF is $3 \times 3$ and $D_{n v f}$ is selected as 50. $S_{0}$ and $S_{1}$ are set to 1.5 and 1 , respectively.

Also, Human eyes feel difficulty on small difference when the object has respectively higher or lower depth level. ${ }^{6} \kappa(x, y)$ is calculated as follow.

$$
\kappa(x, y)=p_{1} \cdot d_{\text {nor }}(x, y)^{2}+p_{2} \cdot d_{\text {nor }}(x, y)+p_{3} .
$$

Here, $p_{1}, p_{2}$, and $p_{3}$ are $4,-4$, and 1.5 , respectively. $d_{n o r}(x, y)$ is a normalized depth value corresponding to center view pixel.

The calculated shifted mean watermark noise is added to each extracted noise bar as a watermark signal. These watermark embedded noise bars are then adjusted to have the shifted mean which is the same value of the reference watermark signal $w_{r}$. Finally, we add these noise bars to the denoised center view to make the watermarked center view. 


\subsection{Detection process}

Detection process is a reverse process of embedding process. When a target image is given, we first extract the noise bars by employing Wiener filter as denoising filter. Then we calculate the mean of each noise bars to estimate the embedded watermark signal. After that, the normalized correlation $Z_{n c}$ between the reference watermark signal $w_{r}$ and the extracted watermark signal $w_{e}$ is calculated. The $Z_{n c}$ is given by

$$
Z_{n c}\left(w_{e}, w_{r}\right)=\frac{w_{e} \cdot w_{r}}{\left|w_{e}\right|\left|w_{r}\right|} .
$$

If the $Z_{n c}\left(w_{e}, w_{r}\right)$ exceeds the threshold $\tau$, we determined the watermark is detected.

\subsection{False positive analysis}

Assuming the central limit theorem holds, $Z_{n c}$ follows Gaussian distribution with mean $\mu_{n c}$,

$$
\mu_{n c}=\mu_{w_{e}} \cdot \mu_{w_{r}}
$$

and standard deviation $\sigma_{n c}$,

$$
\sigma_{n c}=\frac{\left|w_{e}\right| \sigma_{w_{r}}}{\left|w_{e}\right|\left|w_{r}\right|}
$$

Since $w_{r}$ follows Gaussian distribution with zero mean and $w_{e}$ also has zero mean according to the first principle to design the scheme, $\mu_{n c}$ is 0 . Since the number of dimensions of $w_{r}$ is $k$, the magnitude of $w_{r}$ is approximately

$$
\left|w_{r}\right| \approx \sqrt{k} \sigma_{w_{r}}
$$

Thus, the variance of $Z_{n c}$ is

$$
\sigma_{n c}^{2} \approx \frac{1}{k}
$$

Since $Z_{n c}$ follows $N\left(0, \sigma_{n c}^{2}\right)$, the probability of a false positive is

$$
\begin{aligned}
& P_{f p}=\int_{\tau}^{\infty} \frac{1}{\sqrt{2 \pi} \sigma_{n c}} \exp \left(\frac{-x^{2}}{2 \sigma_{n c}^{2}}\right) d x \\
& =\int_{\tau}^{\infty} \frac{1}{\sqrt{\frac{2 \pi}{k}}} \exp \left(\frac{-x^{2}}{\frac{2}{k}}\right) d x \\
& =\int_{\sqrt{k} \tau}^{\infty} \frac{1}{\sqrt{2 \pi}} \exp \left(\frac{-t^{2}}{2}\right) d t \\
& =\operatorname{erfc}(\sqrt{k} \tau) .
\end{aligned}
$$

In this study, the threshold $\tau$ is determined to have a false positive rate $P_{f p}$ of about $10^{-6}$.

\section{EXPERIMENTAL RESULTS}

This section shows experimental results to measure the fidelity and robustness of proposed stereoscopic watermarking scheme. To do this, we used 4 pairs of center and depth images shown in fig. 3 . These image pairs are obtained from Middlebury Stereo Datasets ${ }^{7,8}$ and Microsoft Research 3D Video Datasets. ${ }^{9}$ The resolution $m \times n$ of test center views and depth maps are ranged from $1024 \times 768$ to $1390 \times 1110$, and the dimension of watermark signal $k$ is set to $n / 6$. For DIBR setting, $t_{x}$ is set to $5 \%$ of the image width and $f$ is set to $1 . Z_{\text {far }}$ and $Z_{\text {near }}$ are set to 1 and $t_{x} / 2$, respectively. The variances $c_{0}$ and $c$ of watermark signal and shifted mean watermark noise are both set to 0.2 . 
Table 1. Visual quality results of subjective and objective analysis

\begin{tabular}{c|c|c|c||c|c|c|c}
\hline \multirow{2}{*}{} & \multicolumn{3}{|c||}{ Art } & \multicolumn{3}{c}{ Ballet } \\
\cline { 2 - 7 } & Center view & Left view & Right view & Center view & Left view & Right view \\
\hline MOS & 4.8 & 4.7 & 4.7 & 4.5 & 4.3 & 4.4 \\
\hline PSNR & 47.0585 & 47.1260 & 47.0106 & 43.7225 & 0.9887 & 0.9889 \\
\hline SSIM & 0.9967 & 0.9966 & 0.9967 & 0.9890 & Dolls \\
\hline & \multicolumn{3}{|c||}{ Breakdancer } & Center view view & Right view \\
\cline { 2 - 8 } & Center view & Left view & Right view & 4.7 & 4.7 \\
\hline MSS & 4.5 & 4.5 & 4.4 & 45.9837 & 46.0530 & 45.9963 \\
\hline SSIM & 43.4747 & 43.6297 & 43.6251 & 0.9971 & 0.9971 & 0.9971 \\
\hline
\end{tabular}

\subsection{Fidelity}

To measure the visual quality of proposed scheme, we adopted the double stimulus continuous quality scale (DSCQS) method as a subjective analysis. ${ }^{10}$ The perceived overall quality is rated on a quality categorical scale from 1 (bad) to 5 (excellent) using the criteria of mean opinion score (MOS). Ten expert observers participated in the test. Also, we calculated the PSNR and SSIM between the original image and watermarked image as an objective analysis. The experimental results for fidelity test are shown in table 1. As shown in the table, not only MOSs but also PSNR and SSIM for both 2D center view and 3D warped left, right views are high enough. From the results, we can conclude that the overall visual quality is satisfactory.

\subsection{Robusness}

To prove the robustness of proposed scheme, we compared to Lin's scheme, ${ }^{3}$ which is robust to the $2 \mathrm{D}-3 \mathrm{D}$ conversion, under various environments. In the experiments, the threshold $\tau$ is chosen the value of $\operatorname{erfc}(\sqrt{k} \tau)$ to be $10^{-6}$, and it means that value of $\sqrt{k} \tau$ to be 3.4589. Since $k$ is equal to $\frac{n}{6}$ and $\tau$ is proportional to $\frac{1}{\sqrt{k}}, \tau$ is directly proportional to $\frac{1}{\sqrt{n}}$. More mathematically we have

$$
\tau=\sqrt{\frac{6 v^{2}}{n}}
$$

where $v$ is 3.4589. $\tau$ is calculated as 0.2543 in the cases of Art and Dolls. Likewise, $\tau$ is 0.3057 in the cases of Ballet and Breakdancer.

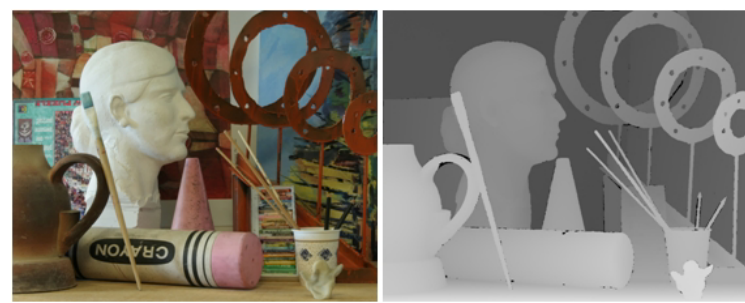

(a) Art $(1390 \times 1110)$

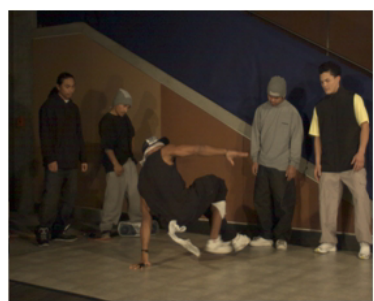

(c) Breakdancer $(1024 \times 768)$
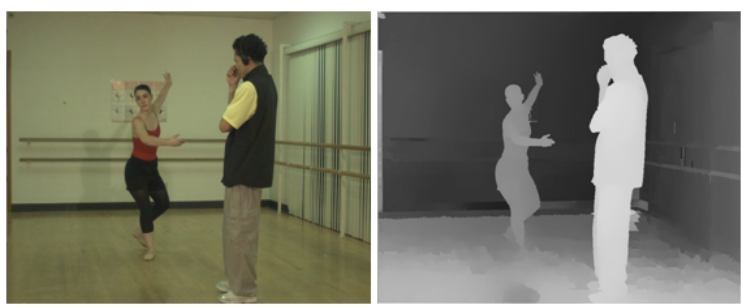

(b) Ballet $(1024 \times 768)$
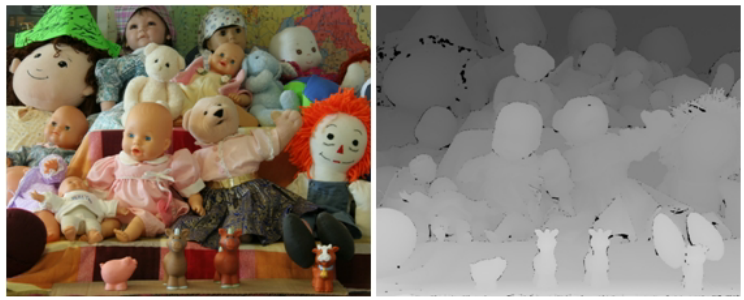

(d) Dolls $(1390 \times 1110)$

Figure 3. Used center views and depth maps 


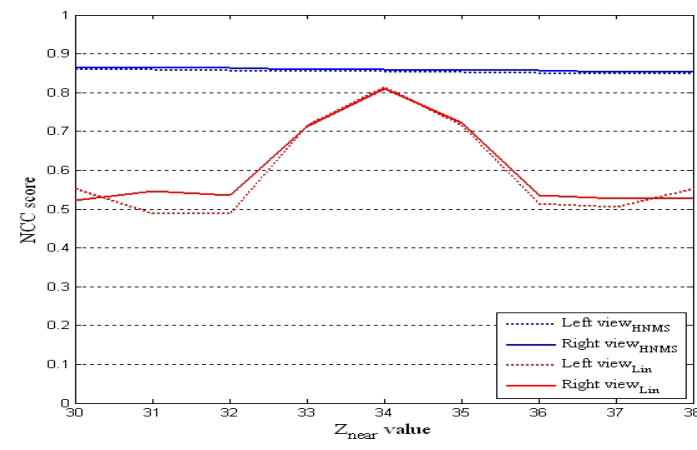

(a) Art (Embedded $\left.Z_{\text {near }}=34\right)$

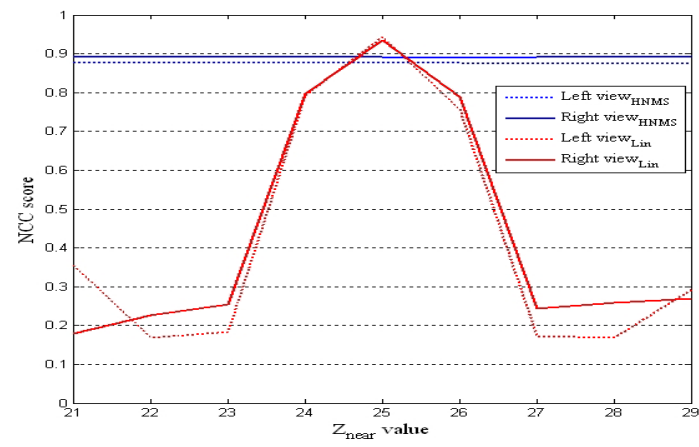

(c) Breakdancer (Embedded $Z_{\text {near }}=25$ )

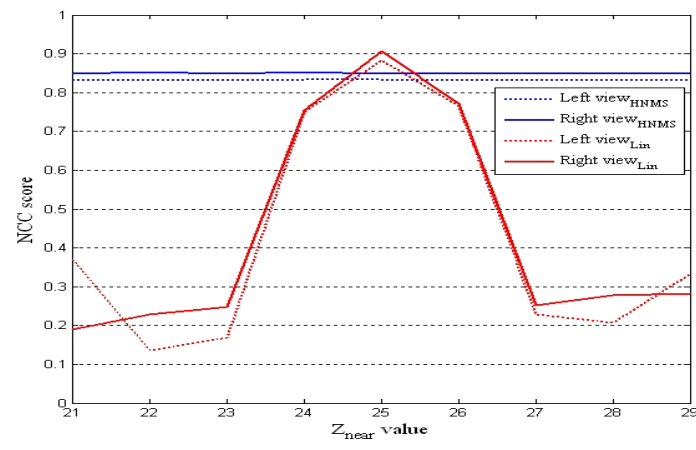

(b) Ballet (Embedded $\left.Z_{\text {near }}=25\right)$

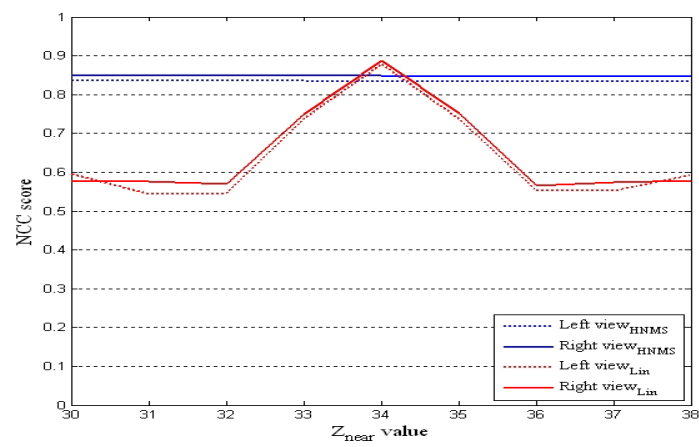

(d) Dolls (Embedded $Z_{\text {near }}=34$ )

Figure 4. $Z_{n c}$ scores of two schemes (HNMS, Lin) when various depth conditions, $Z_{\text {near }}$, are given

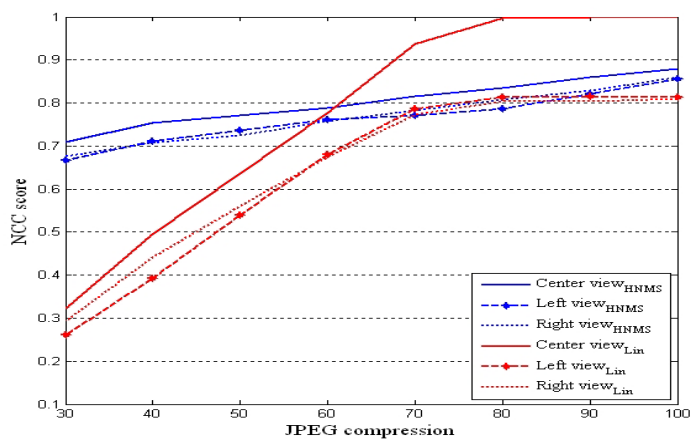

(a) Art

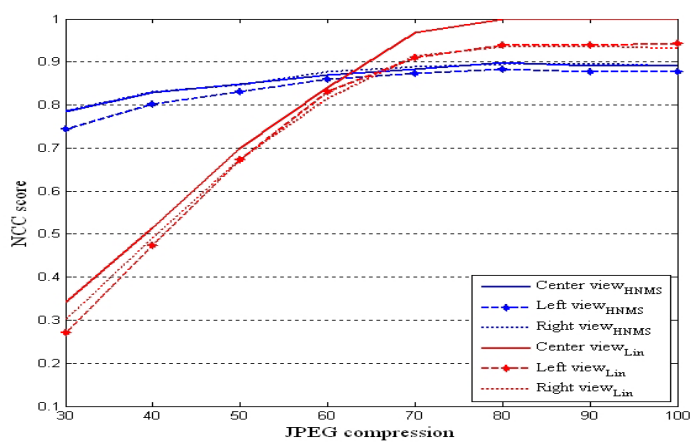

(c) Breakdancer

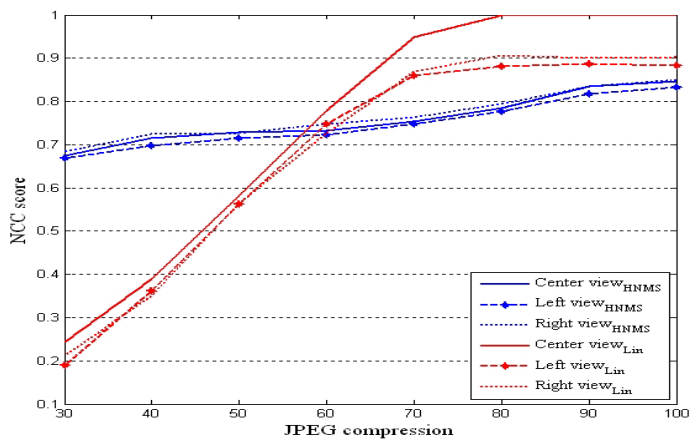

(b) Ballet

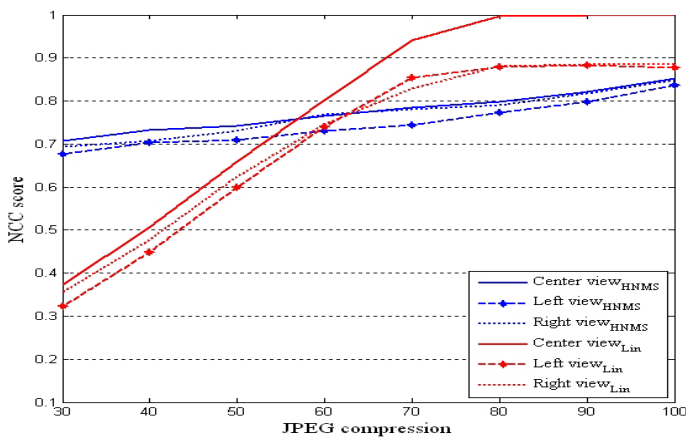

(d) Dolls

Figure 5. $Z_{n c}$ scores of two schemes (HNMS, Lin) when various JPEG compression rates are given 


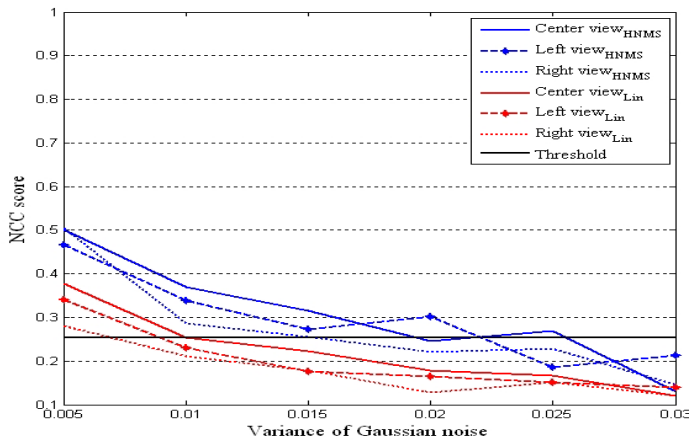

(a) Art

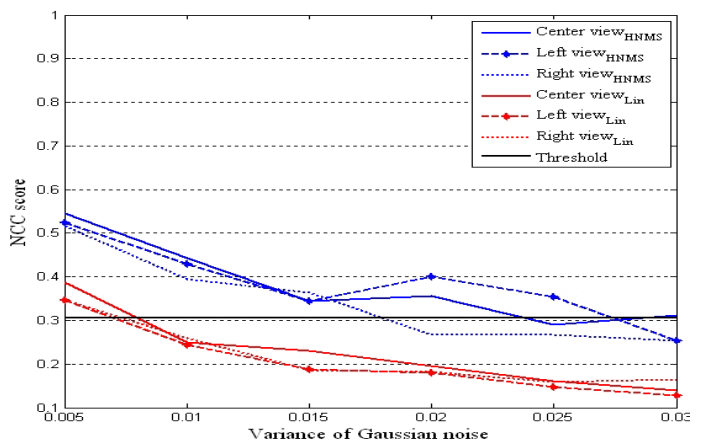

(c) Breakdancer

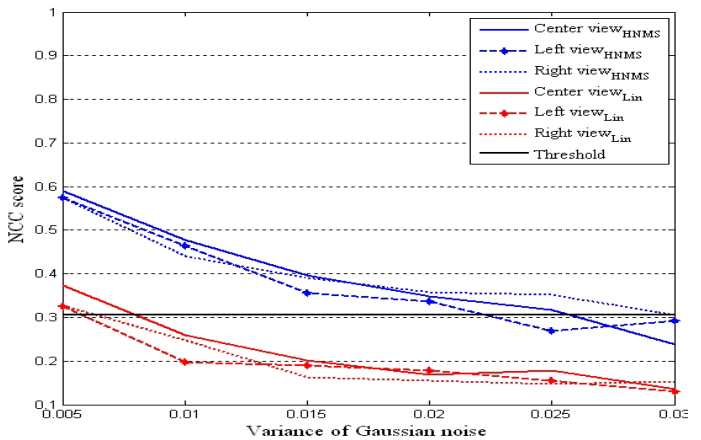

(b) Ballet

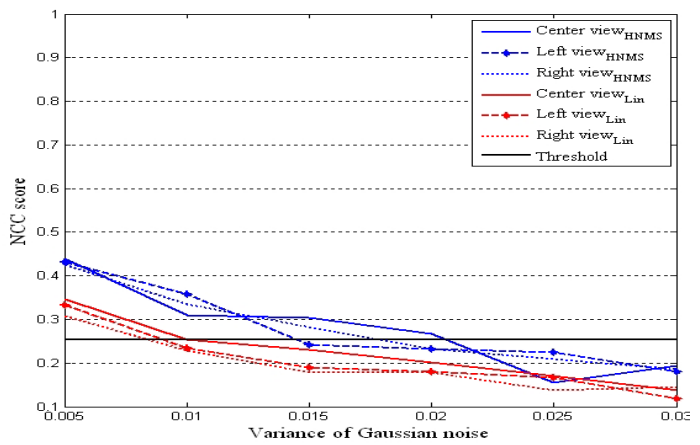

(d) Dolls

Figure 6. $Z_{n c}$ scores of two schemes (HNMS, Lin) when various variances of Gaussian noise are added

Fig. 4 shows the $Z_{n c}$ scores of two schemes (referring to HNMS and Lin, respectively) when the left and right views which were made from various depth conditions by changing the $Z_{\text {near }}$ value were given. Without depth condition, other conditions that may affect the $Z_{n c}$ score were controlled. The aspects of $Z_{n c}$ scores are quite different between the proposed scheme and Lin's scheme when the warped left and right views that have various depth conditions were given. The $Z_{n c}$ scores of Lin's scheme dropped sharply when the difference between embedded $Z_{\text {near }}$ value and warped $Z_{\text {near }}$ value increased gradually. However, the $Z_{n c}$ scores were not

Table 2. $Z_{n c}$ scores of HNMS and Lin when Denoised image is given

\begin{tabular}{|c|c|c|c|c|c|c|c|c|c|}
\hline & \multicolumn{4}{|c|}{ Art } & \multicolumn{4}{|c|}{ Ballet } \\
\hline & & $\begin{array}{l}\text { Mean } \\
3 \times 3\end{array}$ & $\begin{array}{l}\text { Mean } \\
5 \times 5\end{array}$ & $\begin{array}{c}\text { Median } \\
3 \times 3\end{array}$ & $\begin{array}{c}\text { Median } \\
5 \times 5\end{array}$ & $\begin{array}{l}\text { Mean } \\
3 \times 3\end{array}$ & $\begin{array}{l}\text { Mean } \\
5 \times 5\end{array}$ & $\begin{array}{c}\text { Median } \\
3 \times 3\end{array}$ & $\begin{array}{c}\text { Median } \\
5 \times 5\end{array}$ \\
\hline \multirow{2}{*}{$\begin{array}{l}\text { Center } \\
\text { view }\end{array}$} & $\overline{\mathrm{HNMS}}$ & 0.4393 & 0.2601 & 0.6586 & 0.4844 & 0.5666 & 0.4707 & 0.5956 & 0.4894 \\
\hline & Lin & 0.7071 & 0.1703 & 0.7870 & 0.2826 & 0.8779 & 0.5863 & 0.9235 & 0.6344 \\
\hline \multirow{2}{*}{$\begin{array}{l}\text { Left } \\
\text { view }\end{array}$} & HNMS & 0.4124 & 0.2156 & 0.6367 & 0.4195 & 0.5501 & 0.4708 & 0.5694 & 0.5071 \\
\hline & Lin & 0.6113 & 0.1059 & 0.6671 & 0.2278 & 0.7231 & 0.1442 & 0.7739 & 0.2336 \\
\hline \multirow{2}{*}{$\begin{array}{l}\text { Right } \\
\text { viêw }\end{array}$} & HNMS & 0.4085 & 0.2484 & 0.6295 & 0.4700 & 0.5577 & 0.4723 & 0.5946 & 0.5031 \\
\hline & Lin & 0.6311 & 0.1981 & 0.6795 & 0.2825 & 0.7409 & 0.2190 & 0.7916 & 0.3073 \\
\hline & & \multicolumn{4}{|c|}{ Breakdancer } & \multicolumn{4}{|c|}{ Dolls } \\
\hline & & $\begin{array}{c}\text { Mean } \\
3 \times 3\end{array}$ & $\begin{array}{l}\text { Mean } \\
5 \times 5\end{array}$ & $\begin{array}{c}\text { Median } \\
3 \times 3\end{array}$ & $\begin{array}{c}\text { Median } \\
5 \times 5\end{array}$ & $\begin{array}{l}\text { Mean } \\
3 \times 3\end{array}$ & $\begin{array}{l}\text { Mean } \\
5 \times 5\end{array}$ & $\begin{array}{c}\text { Median } \\
3 \times 3\end{array}$ & $\begin{array}{c}\text { Median } \\
5 \times 5\end{array}$ \\
\hline \multirow{2}{*}{$\begin{array}{l}\text { Center } \\
\text { view }\end{array}$} & HNMS & 0.5855 & 0.5322 & 0.6885 & 0.6310 & 0.3828 & 0.2915 & 0.5844 & 0.4501 \\
\hline & Lin & 0.8387 & 0.2003 & 0.9036 & 0.3199 & 0.6539 & 0.1525 & 0.7418 & 0.2745 \\
\hline \multirow{2}{*}{$\begin{array}{l}\text { Left } \\
\text { view }\end{array}$} & HNMS & 0.4552 & 0.4102 & 0.6068 & 0.4907 & 0.3516 & 0.2693 & 0.5572 & 0.4206 \\
\hline & Lin & 0.8103 & 0.1493 & 0.8452 & 0.2429 & 0.6204 & 0.0942 & 0.6890 & 0.2225 \\
\hline \multirow{2}{*}{$\begin{array}{l}\text { Right } \\
\text { vięw }\end{array}$} & HNMS & 0.5972 & 0.5427 & 0.6972 & 0.6349 & 0.3939 & 0.2952 & 0.5892 & 0.4436 \\
\hline & Lin & 0.8300 & 0.2334 & 0.8621 & 0.3216 & 0.6480 & 0.1813 & 0.7065 & 0.2868 \\
\hline
\end{tabular}


affected by changes of depth condition in the case of proposed scheme.

Fig. 5 illustrates the $Z_{n c}$ for applying different JPEG compression ratio to the proposed scheme and Lin's scheme. The $Z_{n c}$ scores for watermarked JPEG compressed images were stable even if JPEG quality was 30. It means the proposed scheme is robust to the compression. However, the $Z_{n c}$ scores of Lin's images dropped drastically when JPEG quality became lower than 60 .

Fig. 6 depicts the impact of adding Gaussian noises with zero mean and different variances to the watermarked image using imnoise function of MATLAB. As shown in the results, the $Z_{n c}$ scores of almost all watermarked view were above the threshold $\tau$ when the variance was 0.015. It is meaningful because PSNR and SSIM values between the original image and Gaussian noise with the variance 0.015 inserted image are around $28.4114 d B$ and 0.7851 respectively, which indicates very poor visual quality.

Since the proposed scheme embeds the watermark signal by adding the shifted mean Gaussian noise, we have to evaluate the robustness of proposed scheme when the suspicious image which employed the denoising filter is given. Two commonly used denoising filters, Mean filter and Median filter, with two different window sizes were used in the denoising process. The experimental results are shown in table 2 . The bold numbers in the table indicate that the $Z_{n c}$ value is below the threshold. The results prove that the proposed scheme has enough robustness against the denoising process. Almost all results of test sets exceeded the threshold and we could extract the embedded watermark signal stably.

\section{CONCLUSION}

The DIBR 3D representation method is getting the spotlight due to its numerous advantages. Two biggest advantages among them are selectiveness of $2 \mathrm{D}-3 \mathrm{D}$ viewing and changeable $3 \mathrm{D}$ depth condition. However, these advantages can be seen as two constraints in the view of copyright protection. In other word, the watermarking scheme for DIBR should have the robustness of 2D-3D conversion and be detectable under various 3D depth conditions. In this study, we proposed the stereoscopic watermarking technique that resolves two constraints the DIBR formatted contents. To protect the copyright of contents, the proposed scheme changes the invariant feature of 2D-3D conversion, the mean of horizontal noise, to embed the watermark signal. By doing this, our scheme could survive the 2D-3D conversion under various depth conditions. Further, the fidelity and robustness of the scheme are good enough as the experimental results proving.

The proposed scheme shows good performance; however, there are a few drawbacks to be resolved. This watermark scheme is tested only to the image sets. However, 2D-3D conversion of video contents is researched widely, and few feasible techniques are already developed. Thus, we need to protect their copyrights by expanding the 3D image stereoscopic watermarking scheme. Also, we have to improve the embedding capacity of proposed scheme. The above considerations will provide the direction for future studies.

\section{ACKNOWLEDGEMENTS}

This research project was supported by Ministry of Culture, Sports and Tourism(MCST) and from Korea Copyright Commission in 2011, WCU (World Class University) program (Project No: R31-30007), and NRL (National Research Lab) program (No. R0A-2007-000-20023-0) under the National Research Foundation of Korea and funded by the Ministry of Education, Science and Technology of Korea.

\section{REFERENCES}

[1] Fehn, C., "Depth-image-based rendering (DIBR), compression and transmission for a new approach on 3DTV," Proc. SPIE 5291, 93-104 (2004).

[2] Lee, M. J., Lee, J. W. and Lee, H. K., "perceptualwatermarking for stereoscopic video using depth information," Proc. IIH-MSP 2011, 81-84 (2011).

[3] Lin, Y. H. and Wu, J. L., "A digital blind watermarking scheme for depth-image-based rendering 3D images," IEEE Trans. on Broadcasting 57(2), 602-611 (2011).

[4] Liang, Z. and Tam, W. J., "Stereoscopic image generation based on depth images for 3D TV," IEEE Trans. on Broadcasting 51(2), 191-199 (2005). 
[5] Voloshynovskiy, S., Herrigel, A., Baumgaertner, N. and Pun, T., "A stochastic approach to content adaptive digital image watermarking," Proc. Information Hiding 1768, 211-236 (2000).

[6] De Silva, D. V. S. X., Ekmekcioglu, E., Fernando, W. A. C. and Worrall, S. T., "Display dependent preprocessing of depth maps based on just noticeable depth difference modeling," IEEE Journal of Selected Topics in Signal Processing 5(2), 335-351 (2007).

[7] Scharstein, D. and Pal, C., "Learning conditional random fields for stereo," Proc. CVPR, 1-8 (2007).

[8] Scharstein, D. and Szeliski, R., "High-accuracy stereo depth maps using structured light," Proc. CVPR, 195-202 (2003).

[9] Zitnick, C., Kang, S., Uyttendaele, M., Winder, S. and Szeliski, R., "High-quality video view interpolation using a layered representation," ACM Trans. on Graphics 23(3), 600-608 (2004).

[10] International Telecommunication Union (ITU) Radio communication Sector, "Methodology for the subjective assessment of the quality of television pictures," ITU-R BT.500-12 (2009). 